\title{
The value of comparative radiographs in the diagnosis of extremity fractures in children by doctors in the emergency department
}

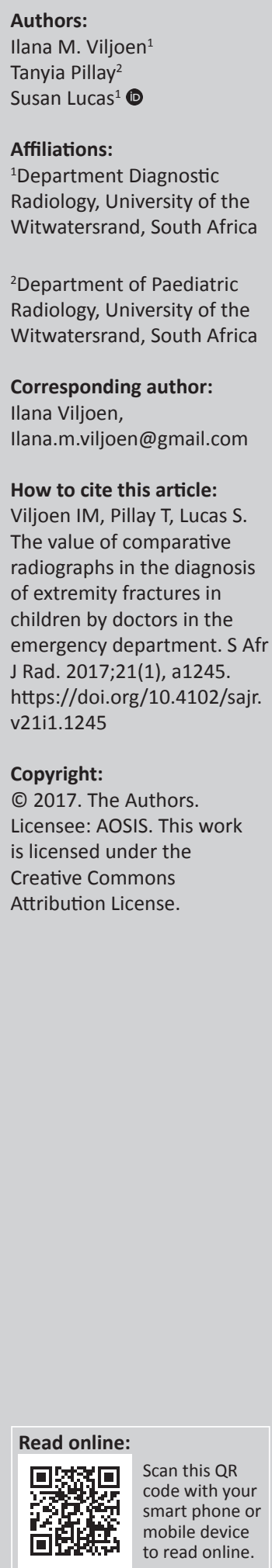

Introduction: The practice of routinely requesting radiographs of both limbs (injured and unaffected side) in a suspected extremity fracture in a child for comparison purposes is widespread and accepted in emergency departments in South Africa, despite the fact that it has been discouraged in literature.

Aim: The aim of this study was to evaluate the ability of junior emergency department doctors to diagnose selected common paediatric extremity fractures without and with comparative radiographs.

Method: Study participants evaluated a set of 30 paediatric extremity radiographs, first without comparison radiographs and then 5 days -7 days later with comparison radiographs. They indicated the presence or absence of a fracture and their level of confidence in making a diagnosis. The selected radiographs included normal films as well as subtle and obvious fractures at the following sites: hand or wrist or forearm, elbow and foot or ankle or lower leg.

Results: The overall ability to detect paediatric extremity fractures (sensitivity) was unchanged $(80 \%)$ with and without comparison radiographs $(p=0.28)$. There was, however, a significant $(7.8 \%)$ increase in detection rates of elbow fractures only, when comparison radiographs were added ( $p=0.0068)$. There was a significant improvement in confidence levels $(12 \%)$ amongst the participants with comparative radiographs compared to without them $(p=0.0001)$. The ability to detect normal paediatric extremity radiographs (specificity) improved by $15.3 \%$ with the addition of comparison radiographs $(p \leq 0.0001)$.

Conclusion: There is an overestimation of the value of comparison radiographs in diagnosing paediatric extremity fractures. The findings of this study do not support their routine use in the emergency department. Selective use is justified. Training of junior emergency medicine doctors to recognise paediatric fractures should be prioritised rather than relying on comparative radiographs.

Note: A selection of conference abstracts: RSSA/SASPI Paediatric Imaging Congress, 03-06 November 2016, Spier Estate, Stellenbosch, South Africa. Faculty collaborators: Professor Kassa Darge (Body Imaging, University of Pennsylvania, Philadelphia, USA), Professor Edward Lee (Thoracic Imaging, Harvard University, USA), Professor Beverley Newman (Cardiac Imaging, Stanford University, California, USA), Professor Kimberly Applegate (Image Gently and Body Imaging, Emory University, Atlanta, USA) and Professor Savvas Andronikou (Thoracic Imaging, University of Bristol, UK) supported by South African Paediatric Radiologists, co-ordinated by Dr Jaishree Naidoo, President of the African Society of Paediatric Imaging and Head of Division of Paediatric Radiology, Charlotte Maxeke Johannesburg Academic Hospital. 